\title{
Bending Tests of Connection Structure of Precast Concrete Slabs using Perfobond Strip
}

\author{
Minh Hai Nguyen $^{1}$, Ryoma Obata ${ }^{1}$, Satoshi Fujiwara ${ }^{2}$, Akinori Nakajima ${ }^{1}$ and Shuichi Fujikura ${ }^{1}$ \\ ${ }^{1}$ Department of Civil engineering and Regional Design, Utsunomiya University \\ Yoto 7-1-2, Utsunomiya City, Japan \\ nguyenminhhai@cc.utsunomiya-u.ac.jp; r169309@cc.utsunomiya-u.ac.jp; \\ akinorin@cc.utsunomiya-u.ac.jp; shuichi.fujikura@cc.utsunomiya-u.ac.jp \\ ${ }^{2}$ Technical Center, D.C Co., Ltd \\ Higashida-cho 8, Kawasaki City, Japan \\ fujiwara_satoshi@dccorp.jp
}

\section{Extended Abstract}

In replacing the slab of girder bridges, a precast concrete slab is often used in order to shorten the construction period on site. In this case, the precast concrete slabs are required to connect together, and conventionally the connection structures with the loop reinforcements [1] or the headed bars [2] are often used. However, it is expected to develop a connection structure with further reduced the dimension and the work-in-site than conventional ones.

This study focuses a new connection structure with the perfobond strip. In this structure, a half part of perfobond steel plates is connected to the edge of the precast slab when producing slabs at the factory, and the other half with two perforations for each plate is arranged into the connection on site. Precast concrete slabs are joined together after filling the cast-in-place the steel fiber reinforced mortar (SFRM) into the connection. Advantages of this method is that, we only arranges a perfobond strip, not arranging any reinforcement into the connection. Therefore, there is no need for the work to arrange reinforcements into the connection on site, or for securing a space for these reinforcements. This allows for smaller dimensions for the connection structure, energy conservation for the on-site work, and shorter construction period on site.

In this study, the bending tests are carried out in order to confirm the bending behaviour of above connection structure. Four specimens that their width and length are $600 \mathrm{~mm}$ and $2200 \mathrm{~mm}$ respectively, are employed. There are two experimental parameters, the first is the volume content steel fibers inside of SFRM using to the connection, and the second is the presence or absence of a chin instead of formwork at the under surface of the connection when casting SFRM on the site. From the tests results, the main findings of this study are summarized as below.

1. Under the action of bending moment, the cracks on the under surface of the connection structure concentrate on the interface between SFRM and the precast concrete. It is considered that the bond strength between concrete and SFRM is smaller than the tensile strength of both.

2. The maximum bending moment of the connection structure is decided on the shear resistance of the perfobond strip, which depends on the restraint effect around the perfobond strip inside the connection.

3. The maximum bending moment of the connection structure increases with the volume content of steel fiber inside of SFRM. The chin instead of formwork at the under surface of the connection, reduces the maximum bending moment of the connection structure. This is because the restraint effect around the perfobond strip decreases when placing a chin or decreasing the volume content of steel fiber inside of SFRM.

4. When the volume content steel fiber inside of SFRM is $2.3 \%$, the maximum bending moment of the connection structure with SFRM at 3 days curing age, is larger about 3 times than the slab design moment.

\section{References}

[1] Hyung-Keun Ryu, Young-Jin Kim and Sung-Pil Chang, "Experimental study on static and fatigue strength of loop joints," Engineering Structures, vol. 29, pp. 145-162, 2007.

[2] Jean Paul Vella, Robert L. Vollum and Andrew Jackson, "Flexural behaviour of headed bar connections between precast concrete panels," Construction and Building Materials, vol. 154, pp. 236-250, 2017. 